\title{
A Smart Dual-Mode Calorimetric Flow Sensor
}

\author{
Vasileios Kitsos, Andreas Demosthenous, Fellow, IEEE, and Xiao Liu, Senior Member, IEEE
}

\begin{abstract}
A smart thermal flow sensor system is presented. It makes use of a novel heater control circuit which can automatically set the operating mode to either constant power or constant temperature difference. It overcomes the limitations of single-mode thermal flow sensors, such as temperature overshoots at low flow rates at constant power mode, or excessive power consumption at high flow rates at constant temperature difference mode. The system is especially useful for temperature sensitive and portable applications, such as respiratory monitoring for medical diagnostics. In this paper, detailed description of the sensor's design, implementation, and experimental validation are presented. The proposed dual-mode flow sensor achieves an overtemperature reduction up to $9.5 \%$ compared with thermal flow sensors operating in constant power mode alone, and a power reduction up to $13.6 \%$ compared with thermal flow sensors operating in constant temperature difference mode alone for the flow range of 0 to $50 \mathrm{slm}$ while offering an improved overall sensitivity.
\end{abstract}

Index Terms - Calorimetric, constant power, constant temperature difference, dual-mode, heater control, multi-modal, thermal flow sensor.

\section{INTRODUCTION}

$\mathrm{F}$ LOW sensors find wide applications in various fields such as in industrial processes [1], meteorology [2][3], and medical diagnostics [4][5][6]. The principle of flow measurement can typically be divided into two main categories: thermal [7][8] and non-thermal flow sensors [9][10][11]. The major drawback of non-thermal topologies is that they can be complex and expensive to fabricate. In contrast, thermal flow sensors make use of heat transfer phenomena to determine the velocity of the fluid. They are structurally and electronically simpler and have no mechanical moving parts. The latter is highly desirable since it offers greater reliability and easier maintenance, while reducing the cost of fabrication. In addition, thermal flow sensors can be fabricated using standard CMOS technologies facilitating the integration with the rest of the electronic circuitry, or using off-the-shelf discrete components mounted on a printed circuit board (PCB), should the physical size of the sensor be less critical.

In general, thermal flow sensors consist of heating elements

This work was supported by Doctoral Training Award from the UK Engineering and Physical Sciences Research Council.

The authors are with the Department of Electronic and Electrical Engineering, University College London, London, WC1E 7JE, United Kingdom (Contact author: xiao@ucl.ac.uk). and temperature sensing elements, and they can be divided into three main sub-categories based on their methods of transduction, namely, hot-wire/hot-film [12][13], calorimetric [14][15], and time-of-flight [16][17]. A time-of-flight flow sensor consists of a heating element producing heat pulses, and a temperature sensing element at a known distance from the heater. The flow rate is determined from the propagation time needed for the heat pulse to reach the temperature sensing element in the known medium. A hot-wire/hot-film flow sensor has a single heating element exposed to the flow. The effect of the flow on hot-wire/hot-film's power consumption or temperature is proportional to the fluid's velocity. Similar to the hot-wire/hot-film, calorimetric flow sensors also have a heating element exposed to the flow. In addition, they have two temperature sensing elements, one at the upstream and one at the downstream, symmetrically placed against the heater. When there is no flow, the heater ideally creates a symmetrical thermal distribution around it, hence, the temperature sensors should measure the same temperature. However, when flow occurs, the thermal distribution is disturbed resulting in a higher temperature at the downstream than at the upstream; thus, the temperature difference $\Delta T$ between the two temperature sensors is a function of the fluid's velocity.

The governing equation for thermal flow sensors' operation and heater's control is derived from King's Law [18]:

$$
\frac{P}{T_{H}-T_{\infty}}=A+B U^{n}
$$

where $P$ is the power consumed by the heater, and $T_{\mathrm{H}}$ and $T_{\infty}$ are the heater's temperature and the ambient temperature of the fluid, respectively. $A, B$, and $n$ are constants that depend on the properties of the fluid and on the characteristics of the materials used for the transducer. The medium's velocity, $U$, is a multi-dimensional parameter, as it is a function of $P, T_{\mathrm{H}}$ and $T_{\infty}$. To simplify flow measurement, it is desirable to reduce $U$ to one dimension by keeping other factors that affect the flow constant. This is translated to operating the heater in one of the following modes: i) a constant $P$ operates the heater in the constant power (CP) mode, ii) a constant $T_{\mathrm{H}}$ operates the heater in the constant temperature (CT) mode, and iii) a constant overtemperature, $T_{\mathrm{OVER}}=T_{\mathrm{H}}-T_{\infty}$, operates the heater in the constant temperature difference (CTD) mode. CP is beneficial from the power management point of view as the exact power consumption is always known. However, the main disadvantage of CP is that the heater's temperature, $T_{\mathrm{H}}$, can rise substantially at zero or low flow rates, leading to potential hazards. For example, heaters made of materials such as platinum are not suitable for high $T_{\mathrm{H}}$ in the presence of flammable gasses [19][20]. Also, increasing $T_{\mathrm{H}}$ at CP mode can 


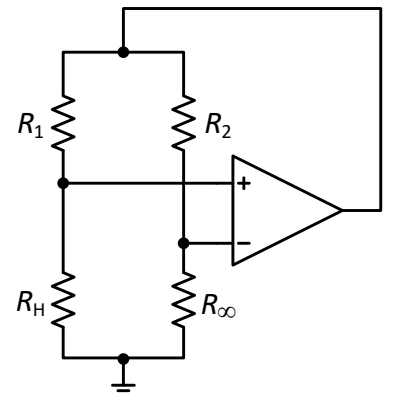

Fig. 1. A Wheatstone bridge used as a heater control circuit in CTD mode. $R_{\mathrm{H}}$ and $R_{\infty}$ are the heating element and the ambient temperature sensing element, respectively.

potentially be dangerous for in-vivo use [21], since it might cause discomfort or even thermal destruction to nearby tissue when the sensor is used for measurement of physiological flows, such as respiration [22], intravascular [4] or, cerebrospinal fluid flow [23]. In contrast, temperature overshoot is not an issue for the CTD as the heater temperature is regulated. However, power consumption can be difficult to predict when the flow is expected to vary substantially, hindering the production of wearable and portable flow measuring devices [24]. Also, excessive power may be needed in high-flow conditions in order to maintain the same overtemperature which might also increase the system's cost. Therefore, CP or CTD mode alone pose significant restrictions to the design of thermally safe, yet portable flow sensors. Operating the heater in the CT mode has also been reported in literature [25]. But it was mainly limited to applications where the ambient temperature, $T_{\infty}$, is constant, or when measurement accuracy is not critical. The CTD offers higher measurement accuracy than the CT as the ambient temperature, $T_{\infty}$, is compensated in the CTD.

The prevalent heater control circuit for CTD is a Wheatstone bridge, as shown in Fig. 1 [26]. Its resistors' values have been carefully selected to establish a desired ratio:

$$
\frac{R_{H}}{R_{\infty}}=\frac{R_{1}}{R_{2}}
$$

where $R_{\mathrm{H}}$ is the heater's resistance, $R_{\infty}$ is the ambient temperature sensing resistor, and $R_{1}$ and $R_{2}$ are resistors of the same temperature coefficient. However, there are two main issues with this topology. Firstly, the branch of $R_{\mathrm{H}}$ requires substantial current in order to operate as a heater, while the $R_{\infty}$ needs minimal current in order to avoid any self-heating that will lead to erroneous measurements of the ambient temperature. This means that $R_{\infty}$ is typically much larger than $R_{\mathrm{H}}$, which might lead to significantly large resistance values, even impractical in case of a miniaturized sensor. Secondly, unless the resistances of $R_{\mathrm{H}}$ and $R_{\infty}$ are exponentially related to their temperature, the Wheatstone bridge establishes a constant temperature ratio rather than a constant temperature difference, adding an error to the measured value. The issue of self-heating has been addressed by Sosna et al. [27] by placing the $R_{\infty}$ in a separate Wheatstone bridge while driven by the same voltage as the bridge of $R_{\mathrm{H}}$. Such a topology has the benefit that low currents at $R_{\infty}$ bridge can be achieved without a large resistance value for $R_{\infty}$, avoiding self-heating. Other solutions such as in

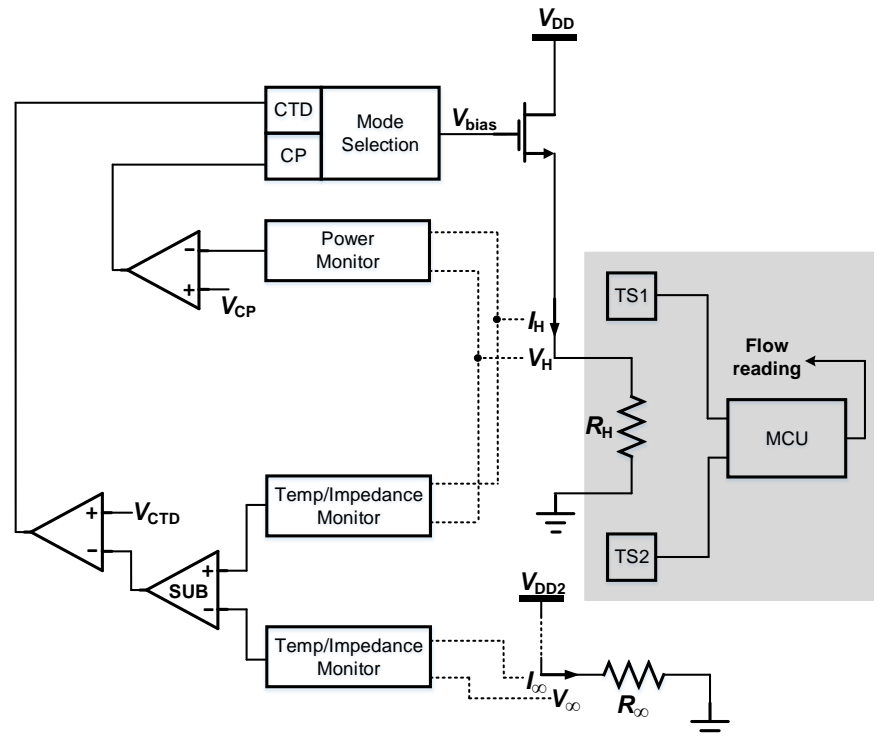

Fig. 2. Functional block diagram of the proposed calorimetric flow sensor. TS1 and TS2 are temperature sensors, and $V_{\mathrm{CP}}$ and $V_{\mathrm{CTD}}$ are reference voltages for the $\mathrm{CP}$ and CTD modes, respectively.

[28] where the resistance of $R_{2}$ continuously switches between two predefined values, $R_{2 \mathrm{x}}$ and $R_{2 \mathrm{y}}$, have also been proposed at the expense of circuit complexity and transient response.

To establish a temperature difference between $T_{\mathrm{H}}$ and $T_{\infty}, \mathrm{Xu}$ et al. [29] introduced a resistor $R_{\mathrm{C}}$ in series with $R_{\infty}$. By setting the value of $R_{C}=a R_{\infty 0} T_{\mathrm{OVER}}$, where $\alpha$ is the temperature coefficient of the $R_{\mathrm{C}}, R_{\mathrm{H}}$ and $R_{\infty}$ resistors, $R_{\infty 0}$ is the nominal resistance of $R_{\infty}$ at its reference temperature, and $T_{\text {OVER }}$ is the intended overtemperature, a temperature difference can be achieved rather a temperature ratio. To the authors' best knowledge, there is not a single heater control circuit for CTD that addresses both issues.

For existing thermal flow sensors operating in $\mathrm{CP}$ mode, the requirement of constant power has been met by either closed-loop control with the aid of a microcontroller (MCU) [30], or with the application of constant current (CC) through the heater [31] or constant voltage (CV) across the heater [32]. However, neither $\mathrm{CC}$ nor $\mathrm{CV}$ leads to a true $\mathrm{CP}$ as the temperature coefficient of $R_{\mathrm{H}}$ leads to erroneous results if not compensated.

This work presents a smart thermal flow sensor which is able to automatically toggle between two different modes, CTD and CP. The former mode operates in low flow rate conditions eliminating the danger of potential hazardous temperature overshoots, whereas CP is activated in high flow rate conditions ensuring a better controlled power budget. The toggling point between the CTD and CP can be selected to suit individual applications. In the literature, one can find thermal flow sensors that have been tested for different operating modes, but there was no linked operation among different modes [33][34]. To the authors' best knowledge, only [35] uses different operating modes for different wind velocities, to create a wind sensor of high accuracy. Nevertheless, [35] requires the temperature sensing elements to switch their operation to heating elements, and the heating elements to temperature sensing elements, every time the operating mode 


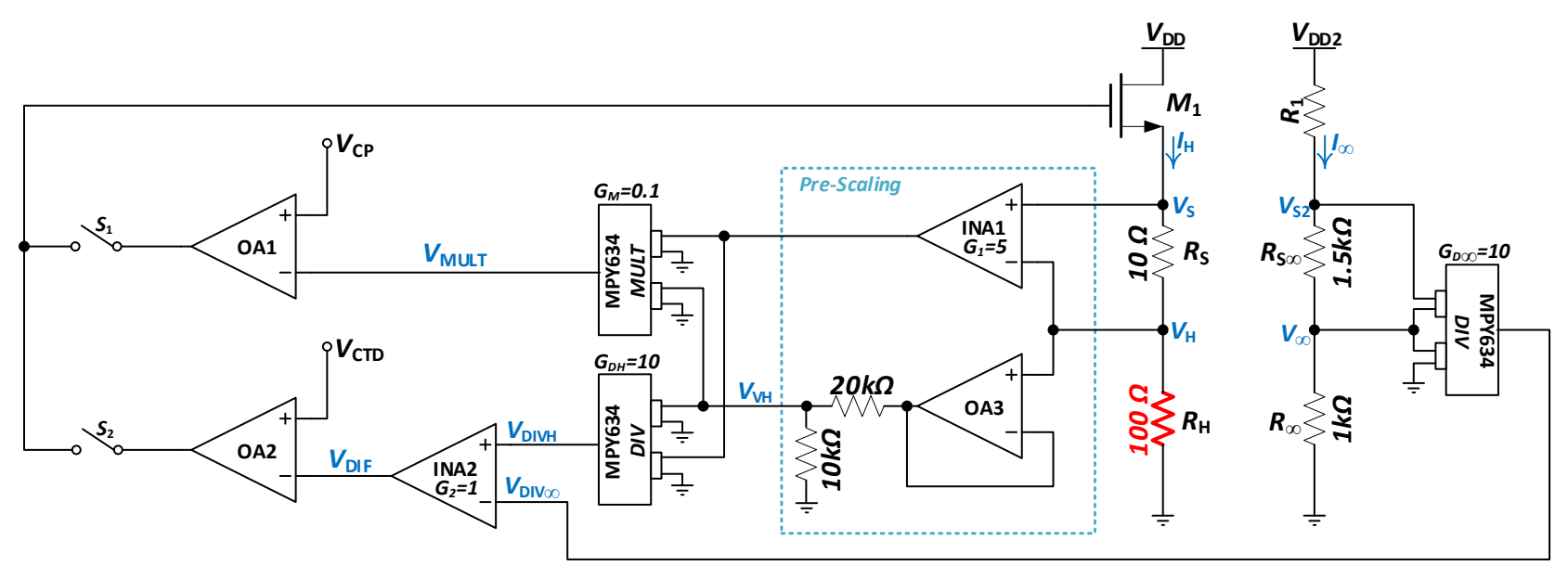

Fig. 3. Simplified schematic of the proposed dual-modal heater control circuit.

changes, compromising the transient response and accuracy of the flow sensor at mode transition. In this work, the heater and sensing elements do not swap their operation, while the toggling point has been chosen in a way that ensures a smooth transition between the modes of operation. The novel dual-mode heater control circuit also addresses common issues found in the literature and described above, i.e. $R_{\infty}$ 's self-heating and the use of temperature ratio for the CTD, and the compensation for the $R_{\mathrm{H}}$ 's resistance variations for the CP mode.

The rest of the paper is organized as follows. Section II describes the architecture of the proposed dual-mode flow sensor, Section III and IV present the experimental setup and measured results, respectively, and Section $\mathrm{V}$ draws conclusions from the presented work.

\section{SYSTEM ARCHITECTURE}

The functional block diagram of the proposed calorimetric thermal flow sensor is shown in Fig. 2. It mainly consists of two sub-systems: the flow measurement circuit (in the shaded area of Fig. 2) and the heater control circuit. For the work presented here, both circuits have been implemented on printed circuit board using discrete components. Nonetheless, miniaturization is feasible using advanced microfabrication and assembly techniques, reducing the system's footprint, overall power consumption and response time [36].

\section{A. Heater Control System}

The flow sensor presented in this paper makes use of two closed-loop heater control circuits, one for the CTD and one for the CP mode, utilizing analog multipliers and analog dividers. Although analog multipliers and dividers have been previously used for either measuring temperature [37] or for maintaining the power constant [38], this the first time used together for creating a smart dual-mode calorimetric flow sensor.

Fig. 3 depicts the proposed heater control circuit schematic. For the CP mode, a low TC resistor $R_{\mathrm{S}}$ is placed in series for measuring the current, $I_{\mathrm{H}}$, through the heater $R_{\mathrm{H}}$. The voltage drop $V_{\mathrm{RS}}$ across $R_{\mathrm{S}}$, and the voltage at the heater $V_{\mathrm{H}}$, are applied to an analog multiplier. Assuming $G_{1}$ is the gain of the instrumentation amplifier INA1, and $G_{\mathrm{M}}$ is the gain of the multiplier, the output of the multiplier $V_{\text {MULT }}$ is:

$$
V_{\mathrm{MULT}}=G_{M}\left(G_{1} V_{R S}\right) V_{H}=\left(G_{M} G_{1} R_{S}\right) I_{H}^{2} R_{H}
$$

Equation (3) states that the output of the multiplier is a linear function of the power at the heater $P=I_{H}^{2} R_{H}$. The accuracy of the two gains $\left(G_{\mathrm{M}}\right.$ and $\left.G_{1}\right)$, as well as the accuracy of the resistance value of the $R_{\mathrm{S}}$, determine the accuracy of the measured power. To achieve a constant power at the heater, the opamp OA1 forms a close-loop control which regulates the gate voltage of the transistor, $M_{1}$, which in turn regulates the heater current.

Fig. 3 also shows the proposed heater control circuit schematic for the CTD mode. Compared to CP, the ambient temperature, $T_{\infty}$, must be also considered; therefore, an additional branch with the $R_{\infty}$ and a low-TC current sensing resistor $R_{\mathrm{S} \infty}$ in series is needed. This topology continuously calculates the resistances of both, $R_{\mathrm{H}}$ and $R_{\infty}$, using analog dividers, and drives the heater's branch based on their difference. Assuming $G_{\mathrm{DH}}$ and $G_{\mathrm{D} \infty}$ are the gains of the dividers for the heater and the ambient temperature sensor, respectively, and $V_{\mathrm{VH}}$ is the heater's voltage, $V_{\mathrm{H}}$, divided by a factor $F$, the output of the dividers $V_{\text {DIVH }}$ and $V_{\text {DIV } \infty}$ are:

$$
V_{\mathrm{DIVH}}=\frac{G_{D H}}{F G_{1} R_{S}} R_{H} \quad \text { and } \quad V_{\mathrm{DIV} \infty}=\frac{G_{D \infty}}{R_{S \infty}} R_{\infty} .
$$

Assuming that the resistances of the $R_{\mathrm{H}}$ and $R_{\infty}$ are a linear function of temperature in the form of:

$$
R=R_{0}\left(1+a\left(T_{X}-T_{R E F}\right)\right)
$$

where $R_{0}$ is the resistance at reference temperature $T_{\mathrm{REF}}$ and $\alpha$ is the temperature coefficient of the resistor, (4) can be re-written as:

$$
\begin{gathered}
V_{\mathrm{DIVH}}=\frac{G_{D H}}{F G_{1} R_{S}} R_{H 0}\left(1+a\left(T_{H}-T_{R E F}\right)\right) \\
V_{\mathrm{DIV} \infty}=\frac{G_{D \infty}}{R_{S \infty}} R_{\infty 0}\left(1+a\left(T_{\infty}-T_{R E F}\right)\right) .
\end{gathered}
$$

The instrumentation amplifier, INA2, monitors the difference 
between $V_{\mathrm{DIVH}}$ and $V_{\mathrm{DIV} \infty}$. By selecting the gains above and the nominal values of the resistors, $R_{\mathrm{H} 0}$ and $R_{\infty}$, in a way such as:

$$
\frac{G_{D H}}{F G_{1} R_{S}} R_{H 0}=\frac{G_{D \infty}}{R_{S \infty}} R_{\infty 0}=G
$$

the output of the instrumentation amplifier, $V_{\mathrm{DIF}}$, will be:

$$
V_{\mathrm{DIF}}=G a\left(T_{H}-T_{\infty}\right) .
$$

Similar to the CP heater control circuit presented above, $V_{\mathrm{DIF}}$ is then applied to the negative input of an opamp, while its positive input is connected to voltage $V_{\mathrm{CTD}}$, corresponding to the desired temperature difference.

Note that after all the different parameters (gains $G_{\mathrm{xx}}, R_{\mathrm{S}}$, $R_{\mathrm{S} \infty}, R_{\mathrm{H} 0}$ and $R_{\infty 0}$ ) have been set, measured, and trimmed if necessary, the topologies presented above can operate in a wide range of power or overtemperature, simply by setting the desirable voltage for $V_{\mathrm{CP}}$ or $V_{\mathrm{CTD}}$, respectively. This feature offers great flexibility, since $V_{\mathrm{CP}}$ and $V_{\mathrm{CTD}}$ may be easily adjusted or changed even on the fly, compared to the Wheatstone bridge where fine adjustments are more difficult to be performed. Also, note that according to (8) the nominal values of the heater and the ambient temperature sensors, $R_{\mathrm{H} 0}$ and $R_{\infty 0}$, do not need to be the same, but have the same temperature coefficient. The proposed heater control circuit better complies with King's Law since it compensates for heater's resistance variations in the $\mathrm{CP}$, whereas it establishes a temperature difference rather than ratio in the CTD mode.

The multi-modal heater control circuit was implemented on a PCB using off-the-shelf components. Three MPY634 chips from Burr-Brown have been used, one as an analog multiplier, and the other two as analog dividers. INA1 has been used as part of the signal pre-scaling. The rest of the pre-scaling is performed by opamp OA3 used as a buffer and the voltage divider created by the $10 \mathrm{k} \Omega$ and $20 \mathrm{k} \Omega$ resistors. The pre-scaling is required to satisfy (8), and to avoid any saturation at the output of the analog multiplier and dividers. Note that the multiplier has an intrinsic gain $G_{\mathrm{M}}$ equal to 0.1 , and the analog dividers $G_{\mathrm{DH}}=G_{\mathrm{D} \infty}=10$, typically accurate to $0.1 \%$. INA1 and INA2 are low-noise INA217 instrumentation amplifiers from Texas Instruments, with gains $G_{1}=5$ and $G_{2}=1$. OA3 is the low input bias current and low-noise opamp OPA4227 from Texas Instruments. The use of a buffer before the voltage divider is preferred to minimize the error caused due to difference in current between the one measured at $R_{\mathrm{S}}$, and the actual current going through the heater $R_{\mathrm{H}}$.

$R_{\mathrm{S}}$ is a $10 \Omega$ SMD chip resistor from Vishay with $\pm 0.1 \%$ tolerance and $\pm 2 \mathrm{ppm} /{ }^{\circ} \mathrm{C}$ temperature coefficient. The voltage divider resistors, as well as the $R_{\mathrm{S} \infty}$, are also SMD resistors with $\pm 0.1 \%$ tolerance and $\pm 5 \mathrm{ppm} /{ }^{\circ} \mathrm{C}$ temperature coefficient. The value of $R_{\mathrm{S} \infty}$ is $1.5 \mathrm{k} \Omega$. $R_{\mathrm{H}}$ and the $R_{\infty}$ are SMD platinum sensing resistors with a temperature coefficient of $3850 \mathrm{ppm} /{ }^{\circ} \mathrm{C}$ from Innovative Sensor Technology (IST), with nominal values of 100 and $1000 \Omega$, respectively. Although the primary function of the IST resistor for $R_{\mathrm{H}}$ is a temperature sensor, it can also operate as a heater without affecting its sensing capability, as long as its temperature does not exceed the maximum temperature rating as specified by the manufacturer.
Based on the values stated above, and by substituting in (3), (8), and (9), the outputs of the multiplier, $V_{\mathrm{MULT}}$, and that of the INA2, $V_{\mathrm{DIF}}$, will be:

$$
V_{\text {MULT }}=\frac{5}{3} P
$$

$$
V_{\mathrm{DIF}}=0.02567 \cdot T_{\mathrm{OVER}} \text {. }
$$

OA1 and OA2 are low-power LM324N opamps from Texas Instruments, and the transistor used is the n-channel power mosfet P16NF06L from STMicroelectronics.

\section{B. Flow Measurement System}

Typically for calorimetric thermal flow sensors, pairs of temperature sensing elements are symmetrically placed next to the heater. Thermistors or thermopiles are commonly used for this purpose. However, their implementation usually requires extra fabrication steps and materials, increasing the fabrication cost. For this work, small digital temperature sensor components have been preferred, specifically a pair of STS21 from Sensirion. The greater size of the IC digital temperature sensor will force the distance between the heater and the actual temperature sensing element to be longer compared to that of a calorimetric micro-machined flow sensor. Nevertheless, the main focus of this work is the heater control systems for $\mathrm{CP}$ and $\mathrm{CTD}$, and their integration to create a smart multi-modal flow sensor, while its flow measurement implementation consists a simple, inexpensive solution.

The flow measurement system has been described in detail in [39]. The readings are acquired using an Arduino Mega micro-controller unit (MCU), a demultiplexer (CD74HC238E) and analog switches (SN74HC4066) from Texas Instruments. The MCU requests from all the sensors simultaneously to take a temperature measurement. The typical time STS21 needs to get a single measurement is $66 \mathrm{~ms}$ and its last reading is automatically stored within the sensor chip. Then, the MCU asks each sensor sequentially to send their saved readings to the MCU. The whole process of measuring and sending the readings to the MCU takes $200 \mathrm{~ms}$ and the sampling frequency is set at $5 \mathrm{~Hz}$. The 14-bit ADC of the STS21 provides a measurement resolution of $0.01{ }^{\circ} \mathrm{C}$.

\section{Mode Switching}

As Fig. 3 shows, analog switches $S_{1}$ and $S_{2}$ are used to toggle between the different modes. Both switches use a MAX14757 chip from Maxim. Either $S_{1}$ or $S_{2}$ can be closed at any given moment and that defines the operating mode. Under either mode during the sensor's operation, the power at the heater, $P$, and the overtemperature, $T_{\mathrm{OVER}}$, are continuously monitored. When the measured $P$ during CTD increases and reaches its threshold value, the state of the switches will swap, activating the CP mode. Similarly, when the flow rate during CP drops enough to reach the maximum allowed $T_{\mathrm{OVER}}$, the switches will swap again and activate the CTD mode. The switches are controlled by the MCU. The threshold values have been chosen in a way that causes minimum disruption at the flow sensor's output, $\Delta T$, offering a smooth change between the modes. 

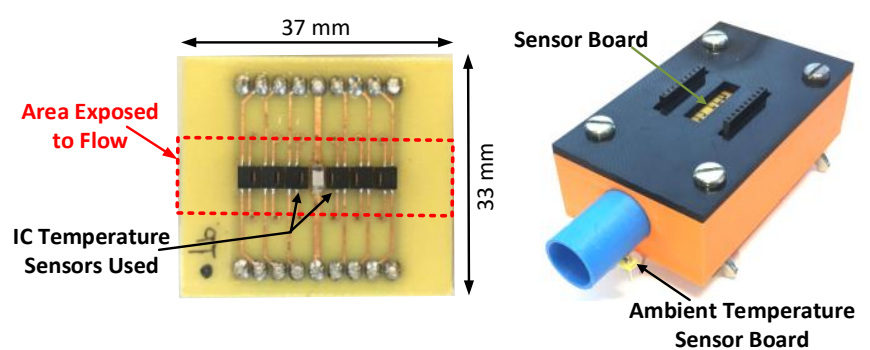

Fig. 4. On left, the sensor where the heating element and the pairs of the digital temperature sensors are mounted. On the right, the 3D-printed housing box where both, the sensor board and the ambient temperature sensor board are mounted.

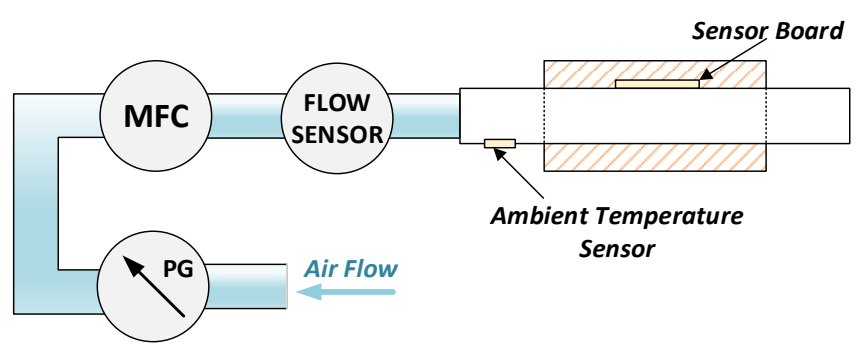

Fig. 5. A cross-sectional drawing of the system's setup. " $P G$ " is the pressure gauge, and "FLOW SENSOR" is the commercial flow sensor from OMRON.

\section{EXPERIMENTAL SETUP}

As Fig. 4 depicts, the heater and the digital temperature sensors (transducer) have been mounted on a $0.8 \mathrm{~mm}$ FR4 PCB, named sensor board. Although three pairs of temperature sensors can be seen in the figure, it is only the pair closest to the heater that has been used for this work. By design, the distance between the edge of the heater and the edge of the temperature sensors (TS) is $0.425 \mathrm{~mm}$. Note that since the copper tracks of PCB might work as heat sinks, the tracks and pins of the board have been placed symmetrically to the central point of the board (where the heater is placed) in order to suppress any possible effect of them on the thermal distribution on the board, and consequently, on the flow measurement.

A 3D printed box has been created to house the sensor board, exposing only the sensing area to the flow. The diameter of the pipe through the housing box is $20 \mathrm{~mm}$. As Fig. 5 depicts, the ambient temperature sensor board is placed diametrically opposite to the heater, and at a horizontal distance of approximately $6 \mathrm{~cm}$, ensuring a thermal isolation between $R_{\mathrm{H}}$ and $R_{\infty}$. The housing box with the boards is then mounted at the end-point of a pipe structure that facilitates the connection to the gas supply. Before the housing box and placed in series, there is the commercially available flow sensor from OMRON (D6F-50A6-000) used as a reference. Before the commercial flow sensor, the mass flow controller (MFC F-201AV) from Bronkhorst is connected to create a programmable flow. The incoming air flow is provided by a pressurized cylinder filled with compressed air. The pressure gauge between the cylinder and MFC regulates the fluid's pressure at 3 bar. The MFC used for this work can create flows in the range of 0 to 50 standard liters per minute $(\mathrm{slm})$ with a resolution of $0.1 \mathrm{slm}$. Note that there is no control over the temperature of the fluid.

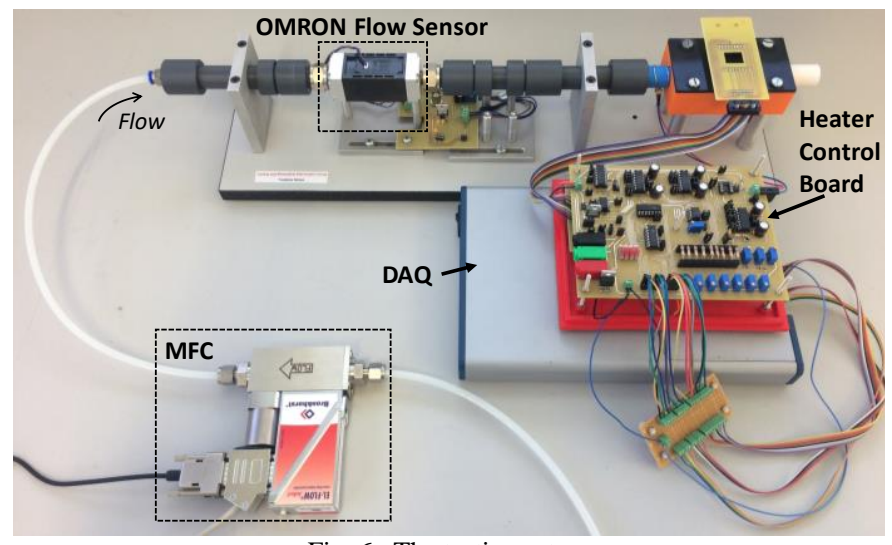

Fig. 6. The testing setup.

National Instruments's USB-6343 data acquisition unit (DAQ) has been used for acquiring the voltage of critical nodes in the heater control circuit and an interactive user interface (UI) has been developed using LabVIEW to allow users to set custom $V_{\mathrm{CP}}$ and $V_{\mathrm{CTD}}$ values subject to the requirements. The DAQ features a 16-bit Digital-to-Analog Converter (DAC), hence, for the output voltage range of $\pm 10 \mathrm{~V}$, the minimum voltage increment is approximately $0.3 \mathrm{mV}$. This voltage increment can be translated into $0.18 \mathrm{~mW}$ minimum power step for the $\mathrm{CP}$, and $0.12{ }^{\circ} \mathrm{C}$ minimum overtemperature step for the CTD. Fig. 6 shows a photo of the testing setup.

The smart flow sensor has been tested for discrete flow values from 0 to $50 \mathrm{slm}$. With the MFC programmed accordingly, the flow remained constant at each flow value for 3 minutes to ensure system stability. The last 250 measurements (equivalent to approximately the last $50 \mathrm{sec}$ ) for each flow value have been taken into account. The maximum and minimum values measured, as well as, the calculated average and standard deviation, are presented. No filtering has been applied to the data presented below.

\section{MEASURED Results}

Fig. 7(a) shows the output of the flow sensor, $\Delta T$, for the three different values of the constant power; 300, 350, and 400 $\mathrm{mW}$. At zero flow, the average heater temperature measured during the experiments, was $95.64,109.82$, and $120.77{ }^{\circ} \mathrm{C}$, respectively. It is apparent that temperatures at heater for $\mathrm{CP}$ mode at no flow conditions can be rather high for temperature sensitive applications such as biomedical, reinforcing the argument for the necessity of multi-modal thermal flow sensors.

The dot points in the Figs. 7(a), (b), and (c), represent the mean values out of the 250 measured for each flow. The error bars that extend from each mean value indicate the absolute maximum and the absolute minimum value measured for each flow rate value, demonstrating the whole range of values acquired during the experiment. To better depict the distribution of the measurements for each flow value, Figs. 7(d), (e), and (f) show their standard deviation for each measurement. A fitting line based on the data has been also created to clearly demonstrate the flow sensor's output performance. 
(a)

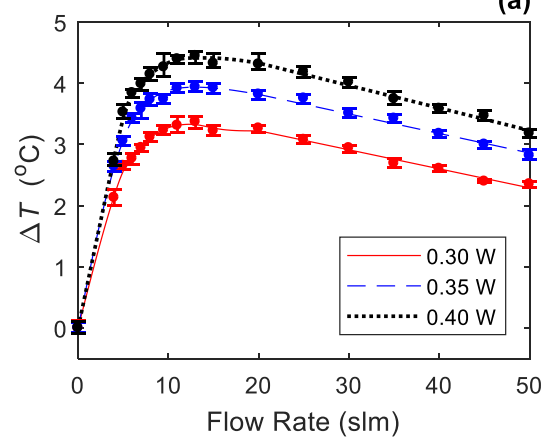

(d)

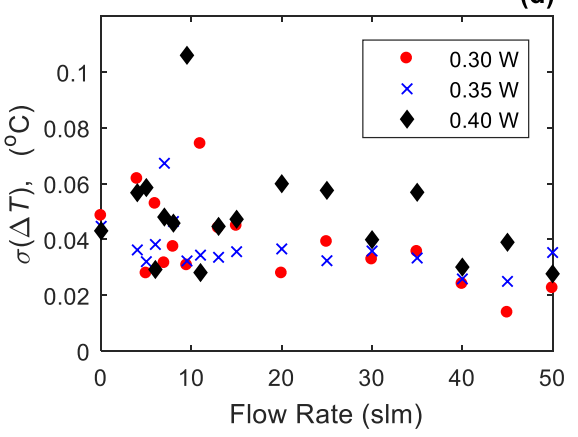

(b)

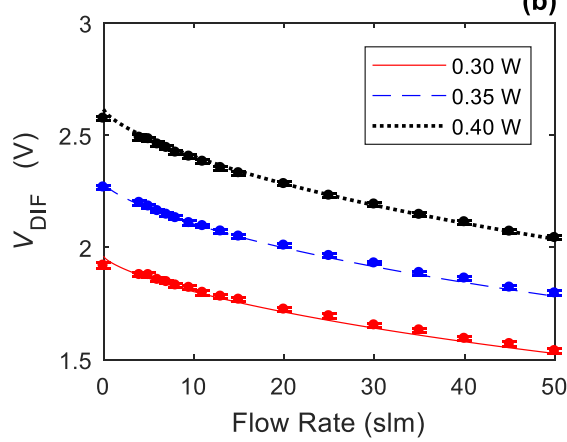

(e)

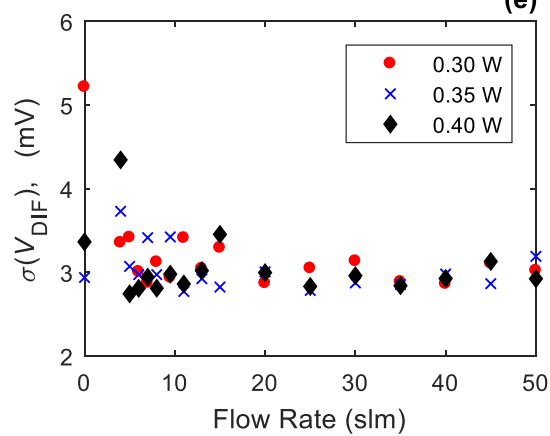

(c)
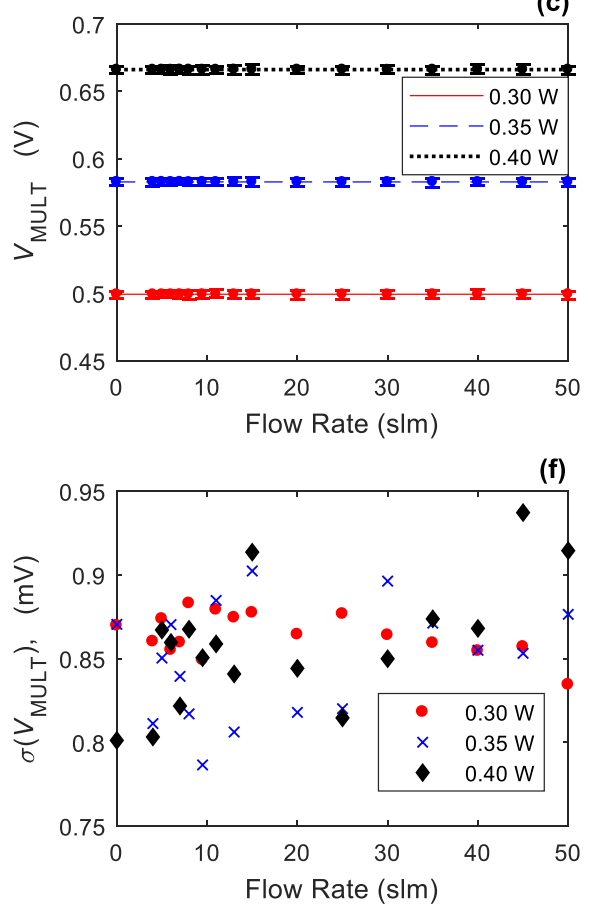

Fig. 7. Experimental results for CP mode. (a), (b), and (c) show the output of the flow sensor as a temperature difference $\Delta T$, the $V_{\mathrm{DIF}}$ that is a function of the $T_{\mathrm{OVER}}$, and the $V_{\text {MULT }}$ that is a function of heater's power consumption, respectively. The second row of graphs corresponds to their standard deviation, $\sigma$.

(a)

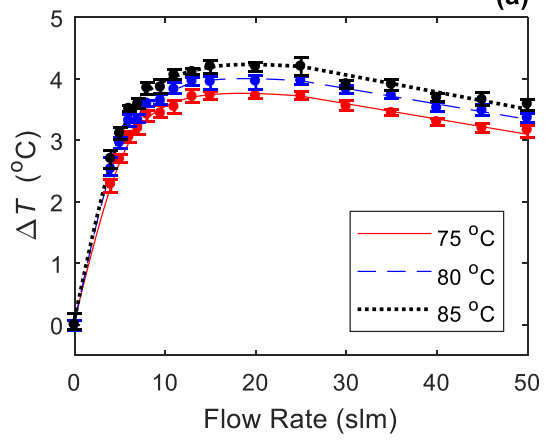

(d)

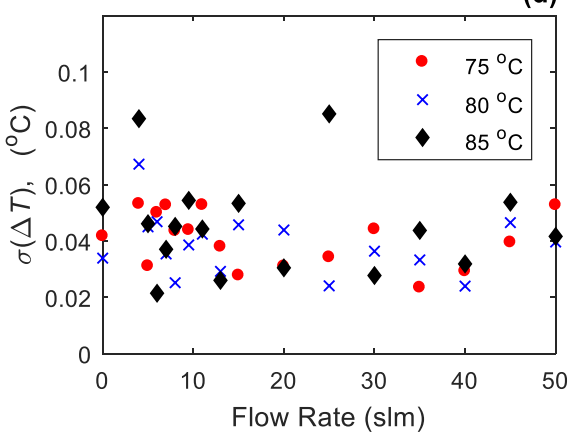

(b)

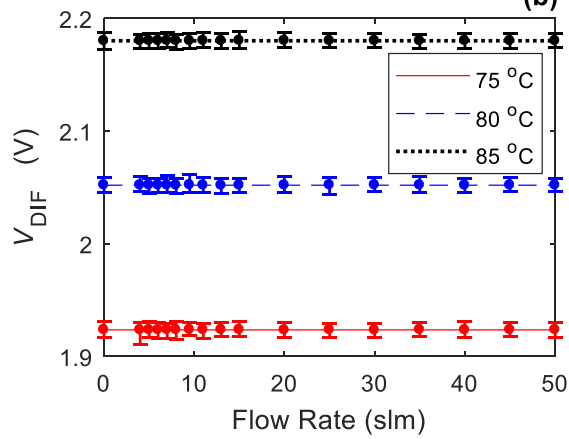

(e)

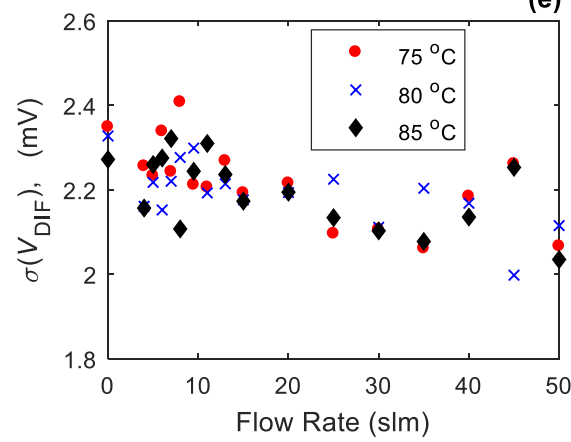

(c)

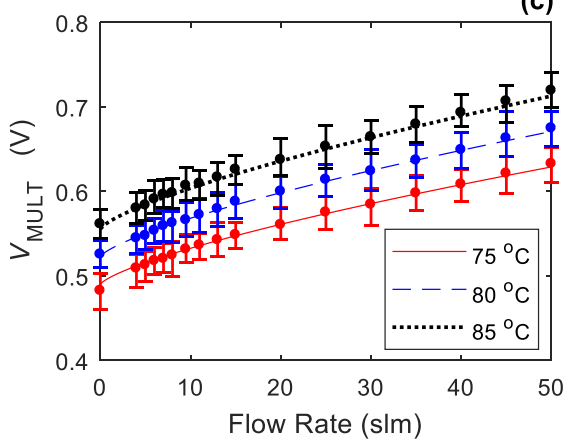

(f)

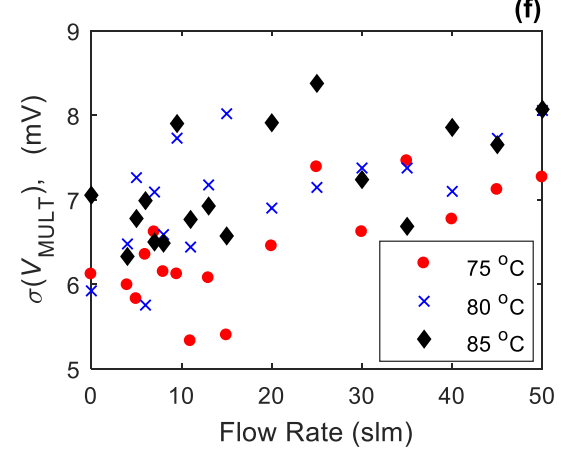

Fig. 8. Experimental results for CTD mode. (a), (b), and (c) show the output of the flow sensor as a temperature difference $\Delta T$, the $V_{\mathrm{DIF}}$ that is a function of the $T_{\mathrm{OVER}}$, and the $V_{\text {MULT }}$ that is a function of heater's power consumption, respectively. The second row of graphs corresponds to their standard deviation, $\sigma$.

In $\mathrm{CP}$ mode, the output increases quickly for low flow rates until it reaches its maximum, also known as turn-over flow rate,

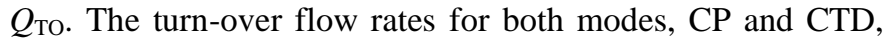
have been calculated and presented in Table I. For flow rates, $Q$, greater than $Q_{\mathrm{TO}}, \Delta T$ slowly reduces, almost linearly. Qualitatively, that is a rather typical output of a calorimetric flow sensor in CP mode.

Figs. 7(b) and (c) depict the voltage related to the overtemperature, $V_{\mathrm{DIF}}$, and the voltage that corresponds to the heater's power, $V_{\text {MULT }}$, respectively. It is demonstrated that the proposed system is capable of keeping the power at the heater constant under any flow and heater temperature conditions 
(a)
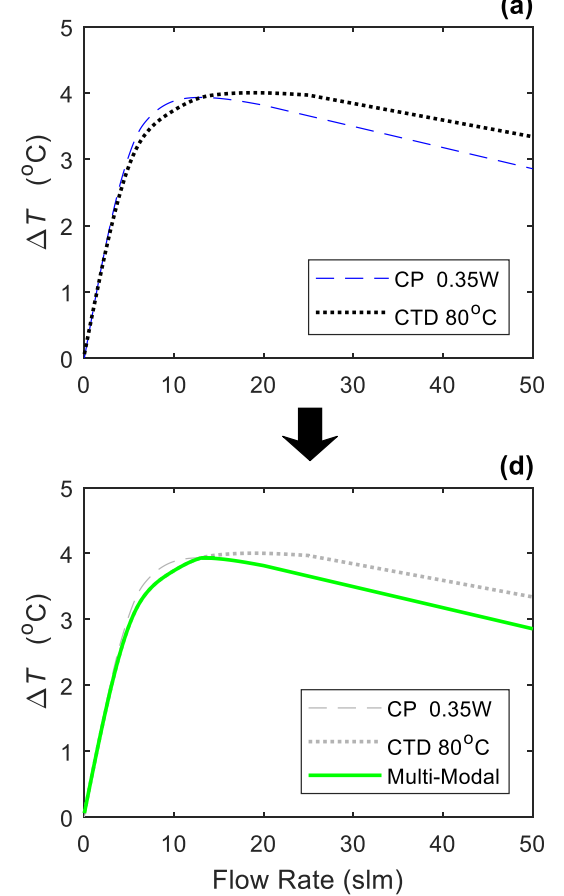

(b)

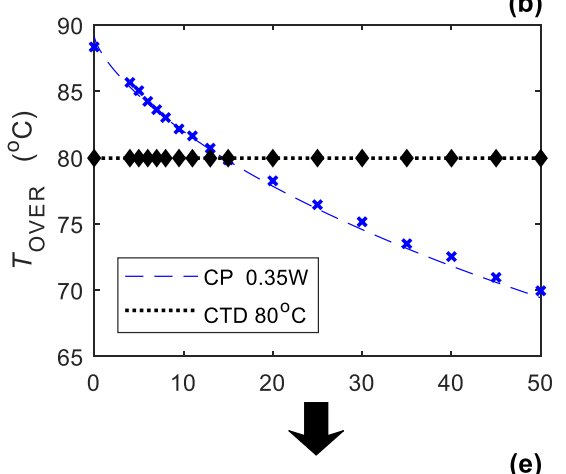

(e)

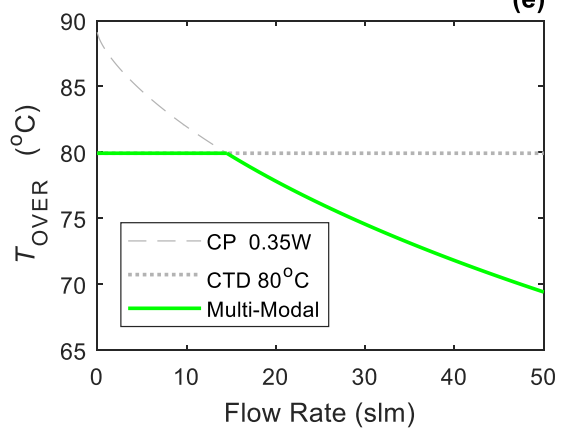

(c)

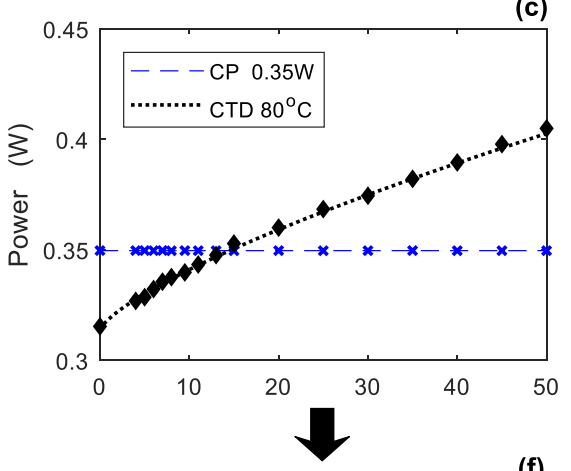

(f)

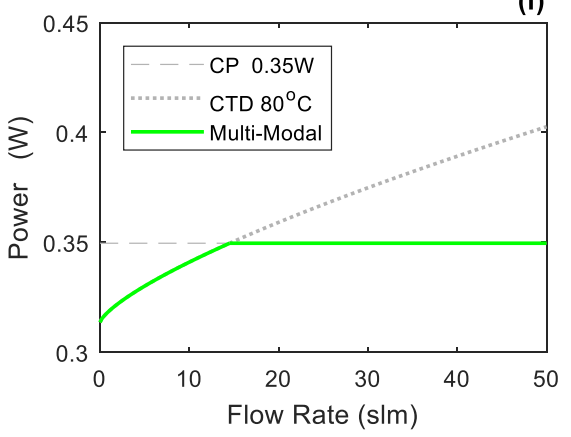

Fig. 9. (a) the flow sensor's output, (b) overtemperature, and (c) heater's power consumption for $\mathrm{CP}$ in $350 \mathrm{~mW}$ and $\mathrm{CTD}$ in $80{ }^{\circ} \mathrm{C}$. At the bottom row, the performance of the multi-modal flow sensor, respectively.

tested. In fact, considering the standard deviation presented, the relative error for the $V_{\text {MULT }}$ is $0.17,0.15$, and $0.13 \%$ for $P$ equal to $0.3,0.35$, and $0.4 \mathrm{~W}$, respectively. $V_{\mathrm{DIF}}$ decreases as the flow rate increases, as expected and according to King's Law in (1).

Fig. 8 shows experimental results for the CTD in a similar format to that used for the $\mathrm{CP}$ mode in Fig. 7, and for overtemperatures of 75,80 , and $85{ }^{\circ} \mathrm{C}$. The output of the flow sensor for the CTD is qualitatively similar to that of the CP mode, where it quickly increases for low flow rates until it reaches a turn-over flow rate, and eventually starts decreasing. However, the CTD outputs seems to have a wider flow rate range of saturation around the turn-over flow rate. This could be a major drawback if one wants to use a continuous measurement range from zero until a flow rate larger than the $Q_{\text {TO}}$, such as the one presented in this work. However, the $Q_{\text {Tо }}$ for the CTD experiments occurs in values higher than those of the $\mathrm{CP}$, as presented in Table I. The measurement range of the flow sensor is greater in the CTD than in CP mode, if only the part from zero to turn-over flow rate is to be used. Such a preference is popular in the literature [40][41]. Note that as the power at the heater increases for a certain mode, either $\mathrm{CP}$ or $\mathrm{CTD}$, the turn-over flow rate increases, too. Hence, the measurement range can be increased by increasing the power consumption, when the part $0 \sim Q_{\text {TO }}$ is only used.

Figs. 8(b), (c), (e), and (f) show the $V_{\mathrm{DIF}}$ and $V_{\mathrm{MULT}}$, and the standard deviation of the measurements. The $V_{\text {DIF }}$ plot demonstrates that the proposed circuit can provide a constant overtemperature under any flow and temperature conditions created during the experiments. This can be supported by the low variation measured and the small $\sigma$. In fact, the relative error of the $V_{\mathrm{DIF}}$ for all the CTD test was always below $0.125 \%$. $V_{\text {MuLt }}$ seems to follow the King's Law, as expected, in (1).
TABLE I

TURN-OVER FLOW RATES $Q_{\text {TO }}$

\begin{tabular}{|c|c|c|c|c|c|}
\hline & & $Q_{\mathrm{TO}}(\operatorname{slm})$ & & & $Q_{\mathrm{TO}}(\operatorname{slm})$ \\
\hline \multirow{3}{*}{$\hat{v}$} & $0.30 \mathrm{~W}$ & 12.4 & \multirow{3}{*}{ 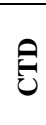 } & $75^{\circ} \mathrm{C}$ & 18.4 \\
\hline & $0.35 \mathrm{~W}$ & 13.0 & & $80^{\circ} \mathrm{C}$ & 18.8 \\
\hline & $0.40 \mathrm{~W}$ & 14.2 & & $85^{\circ} \mathrm{C}$ & 19.0 \\
\hline
\end{tabular}

TABLE II

PARAMETERS FOR FLOWS $Q<Q_{\text {TO }}$

\begin{tabular}{|c|c|c|c|c|}
\hline & & $S^{*}\left({ }^{\circ} \mathrm{C} \mathrm{slm}^{-1}\right)$ & $P(\mathrm{~mW})$ & $T_{\text {OVER }}\left({ }^{\circ} \mathrm{C}\right)$ \\
\hline \multirow{3}{*}{ ชิ } & $0.30 \mathrm{~W}$ & 0.5299 & 300 & $75.76 \sim 69.28$ \\
\hline & $0.35 \mathrm{~W}$ & 0.6113 & 350 & $88.33 \sim 80.59$ \\
\hline & $0.40 \mathrm{~W}$ & 0.7046 & 400 & $100.28 \sim 91.56$ \\
\hline \multirow{3}{*}{ 它 } & $75^{\circ} \mathrm{C}$ & 0.54 & $289.5 \sim 334.1$ & 75 \\
\hline & $80^{\circ} \mathrm{C}$ & 0.5923 & $315.3 \sim 357.1$ & 80 \\
\hline & $85^{\circ} \mathrm{C}$ & 0.6218 & $336.8 \sim 379.7$ & 85 \\
\hline
\end{tabular}

$S^{*}$ : maximum output sensitivity

TABLE III

PARAMETERS FOR FLOWS $Q>Q_{\text {TO }}$

\begin{tabular}{ccccc}
\hline \hline & & $S^{* *}\left|{ }^{\circ} \mathrm{C} \mathrm{slm}{ }^{-1}\right|$ & $P(\mathrm{~mW})$ & $T_{\text {OVER }}\left({ }^{\circ} \mathrm{C}\right)$ \\
\hline \multirow{2}{*}{} & $\mathbf{0 . 3 0} \mathbf{~ W}$ & 0.0311 & 300 & $69.28 \sim 59.93$ \\
& $\mathbf{0 . 3 5} \mathbf{~ W}$ & 0.0319 & 350 & $80.59 \sim 69.94$ \\
& $\mathbf{0 . 4 0 ~ \mathbf { ~ }}$ & 0.0367 & 400 & $91.56 \sim 79.56$ \\
\hline \multirow{2}{*}{ 己 } & $\mathbf{7 5}^{\circ} \mathbf{C}$ & 0.0249 & $334.1 \sim 379.6$ & 75 \\
& $\mathbf{8 0}^{\circ} \mathbf{C}$ & 0.0251 & $357.1 \sim 404.9$ & 80 \\
& $\mathbf{8 5}^{\circ} \mathbf{C}$ & 0.0278 & $379.7 \sim 431.6$ & 85 \\
\hline \hline
\end{tabular}

$S^{* *}:$ maximum output sensitivity in absolute value 

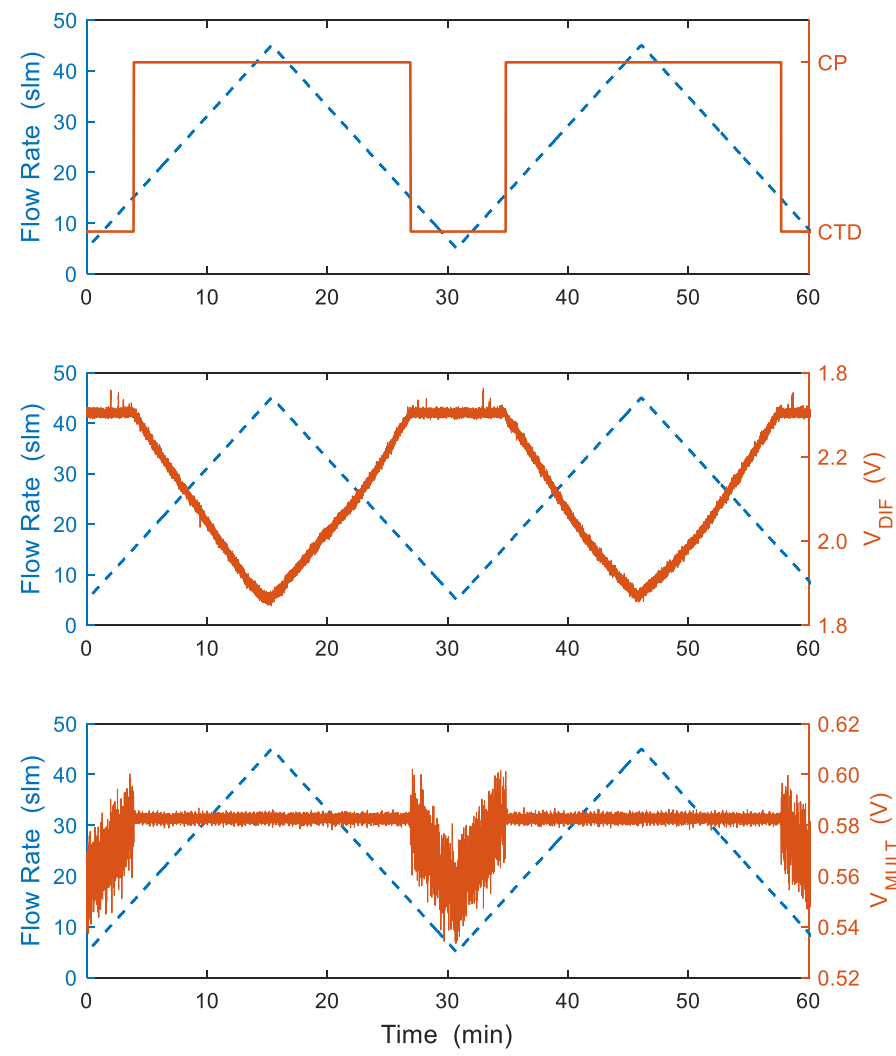

Fig. 10. The top figure states the operating mode over time for a triangular input flow rate. The middle and bottom plots depict the voltages measured for the $T_{\mathrm{OVER}}\left(V_{\mathrm{DIF}}\right)$ and for the $P\left(V_{\mathrm{MULT}}\right)$, respectively, for the same time period.

Heater power measurements in Fig. 8(c) show some variation during a constant flow rate; however, the goal is to achieve a constant overtemperature, hence, fluctuations in power are not important in CTD mode as long as a stable $T_{\text {OVER }}$ is achieved.

Tables II and III summarize the power ratings and the overtemperatures measured before and after the turn-over flow rate. As expected, the greater the power, the better the sensitivity for the CP mode. Similarly, the greater the overtemperature, the better the sensitivity for the CTD mode. This is applicable for both parts of the flow rate range, before and after the $Q_{\text {Tо }}$. However, note that even though the power consumption is greater for CTD than CP for $Q>Q_{\mathrm{TO}}$, the sensitivity is better in the $\mathrm{CP}$. Thus, $\mathrm{CP}$ is the preferred operating mode for flow rates higher than the $Q_{\text {TO }}$. On the other hand, it has been also shown that $\mathrm{CP}$ at low flow rates can be potentially hazardous for temperature sensitive applications. Those findings further support the demand for a smart multi-modal flow sensor.

The top row of graphs of Fig. 9 presents the flow sensor's output $\Delta T$, the overtemperature $T_{\text {OVER }}$ in ${ }^{\circ} \mathrm{C}$, and the power consumed by the heater $P$ for the case of CTD with $80^{\circ} \mathrm{C}$ overtemperature and the case of $\mathrm{CP}$ mode with $0.35 \mathrm{~W}$ heater power. This figure illustrates the differences between the two modes, as experimentally described above. The overtemperature is up to $8{ }^{\circ} \mathrm{C}$ greater for $\mathrm{CP}$ in low flows, whereas the power consumption is almost $55 \mathrm{~mW}$ higher for the CTD, without significantly improving the flow sensor's performance in any other aspect.
Note that in Fig. 9 there is a flow rate value where both, the power at the heater and the overtemperature, are the same for both modes, hence, the output $\Delta T$ is the same. By choosing that flow rate point as the toggling point (the point where the sensor switches from CTD to $\mathrm{CP}$, and vice versa), one can ensure a smooth transition from one mode to the other. The threshold power value that triggers the mode switching from CTD to $\mathrm{CP}$ is the $0.35 \mathrm{~W}$, while the threshold overtemperature value that activates the CTD during $\mathrm{CP}$ operation is set to $80{ }^{\circ} \mathrm{C}$. No abrupt changes in power delivered to the heater need to take place during mode toggling, ensuring a predictable sensor's output.

The bottom row of Fig. 9 shows the behavior of the dual-mode flow sensor. For the measurement results presented here, one can achieve overtemperature reduction up to $9.5 \%$ by using CTD for low flows instead of $\mathrm{CP}$, and heater power reduction up to $13.6 \%$ by using $\mathrm{CP}$ for high flow rates instead of CTD, while improving the sensor's overall sensitivity at the same time. Although the performance improvements are derived by direct comparison between single and dual-mode operation of the same flow sensor in PCB, it may be deduced that similar improvements can be achieved for any calorimetric flow sensor, such as a micromachined flow sensor, that uses dual-modality instead of single.

The transient performance of the smart dual-mode flow sensor is shown in Fig. 10. For a varying input flow rate between 5 and $45 \mathrm{slm}$, the proposed flow sensor can automatically switch between CP and CTD based on the preset threshold values. Fig. 10 shows that as the flow rate increases, the operating mode moves from CTD (constant $V_{\mathrm{DIF}}$ ) to $\mathrm{CP}$ mode (constant $V_{\text {MULT }}$ ). Whereas, the operating mode switches back from $\mathrm{CP}$ to $\mathrm{CTD}$, when the flow rate returns from high to low.

\section{CONCLUSION}

A smart calorimetric flow sensor has been presented. It features a novel heater control system that enables the flow sensor to operate in either CP or CTD. Compared to other work [30][33][42], this sensor can toggle between those modes based on the flow rate measured. The toggling point has been determined as the point where the two modes' outputs are equal, and its choice has been justified based on smooth transition from one to the other. This implementation is capable of producing reproducible results for a large range of flow rates. A quantitative analysis and comparison between the $\mathrm{CP}$ and CTD has been presented, demonstrating the pros and cons of each mode for flows higher and lower than the turn-over flow rate. In fact, this analysis reinforced the requirement for a dual-mode flow sensor, since CP offers a way better performance in high flows than the CTD, whereas the latter still offers an important overtemperature protection in low flow rates. The dual-mode operation demonstrating the automatic switching between the modes has been displayed. The measurements show that any calorimetric flow sensor can save power and reduce overtemperatures by adopting a dual-mode operation, compared to operation in a single mode throughout the measurement range; that is an important improvement 
towards the development of portable and safe thermal flow sensors for biomedical or industrial applications.

\section{REFERENCES}

[1] K. Palmer, H. Kratz, H. Nguyen, and G. Thornell, “A highly integratable silicon thermal gas flow sensor," J. Micromechanics Microengineering, vol. 22 , no. 6, p. 065015,2012

[2] H. B. Liu, N. Lin, S. S. Pan, J. Miao, and L. K. Norford, "High sensitivity, miniature, full 2-D anemometer based on MEMS hot-film sensors," IEEE Sens. J., vol. 13, no. 5, pp. 1914-1920, 2013.

[3] Y.-Q. Zhu, B. Chen, M. Qin, and Q.-A. Huang, "2-D Micromachined Thermal Wind Sensors - A Review," IEEE J. Internet Things, vol. 1, no. 3, pp. 216-232, 2014.

[4] T. Rui, H. Hai, Y. Yong Mo, J. Oiler, L. Mengbing, and Y. Hongyu, "Three-Dimensional Flexible Thermal Sensor for Intravascular Flow Monitoring," IEEE Sens. J., vol. 13, no. 10, pp. 3991-3998, 2013.

[5] F. Hedrich, K. Kliche, M. Storz, S. Billat, M. Ashauer, and R. Zengerle, "Thermal flow sensors for MEMS spirometric devices," Sensors Actuators A Phys., vol. 162, no. 2, pp. 373-378, 2010.

[6] V. Kitsos, S. West, A. Demosthenous, and X. Liu, "Design Considerations and Optimization of Calorimetric Flow Sensor for Respiratory Monitoring," in IEEE Biomedical Circuits and Systems Conference (BioCAS), 2016, no. 2, pp. 26-29.

[7] J. T. W. Kuo, L. Yu, and E. Meng, "Micromachined thermal flow sensors-A review," Micromachines, vol. 3, pp. 550-573, 2012.

[8] C. H. Wu, D. Kang, P. H. Chen, and Y. C. Tai, "MEMS thermal flow sensors," Sensors Actuators A Phys., vol. 241, pp. 135-144, 2016.

[9] J. C. Lötters, T. S. J. Lammerink, J. Groenesteijn, J. Haneveld, and R. J. Wiegerink, "Integrated Thermal and Microcoriolis Flow Sensing System with a Dynamic Flow Range of More Than Five Decades," Micromachines, vol. 3, no. 1, pp. 194-203, Mar. 2012.

[10] P. Enoksson, G. Stemme, and E. Stemme, "A silicon resonant sensor structure for coriolis mass-flow measurements," IEEE J. Microelectromechanical Syst., vol. 6, no. 2, pp. 119-125, 1997.

[11] Q. Zhang, W. Ruan, H. Wang, Y. Zhou, Z. Wang, and L. Liu, “A Self-Bended Piezoresistive Microcantilever Flow Sensor for Low Flow Rate Measurement," Sensors Actuators A Phys., vol. 158, no. 2, pp. $273-$ 279, 2010.

[12] M. M. Sadeghi, R. L. Peterson, and K. Najafi, "Air flow sensing using micro-wire-bonded hair-like hot-wire anemometry," J. Micromechanics Microengineering, vol. 23, p. 085017, 2013.

[13] R. J. Adamec and D. V. Thiel, "Self Heated Thermo-Resistive Element Hot Wire Anemometer," IEEE Sens. J., vol. 10, no. 4, pp. 847-848, Apr. 2010.

[14] W. Xu, K. Song, S. Ma, B. B. Gao, Y. Chiu, and Y.-K. Lee, "Theoretical and Experimental Investigations of Thermoresistive Micro Calorimetric Flow Sensors Fabricated by CMOS MEMS Technology," IEEE J. Microelectromechanical Syst., vol. 25, no. 5, pp. 954-962, 2016.

[15] W. Xu, B. Wang, M. Duan, M. Ahmed, A. Bermak, and Y.-K. Lee, “A 3D Integrated Micro Calorimetric Flow Sensor in CMOS MEMS Technology," IEEE Sensors Lett., vol. 3, no. 2, pp. 1-4, Feb. 2019.

[16] C. Offenzeller, M. Knoll, T. Voglhuber-Brunnmaier, M. A. Hintermuller, B. Jakoby, and W. Hilber, "Fully Screen Printed Thermocouple and Microheater Applied for Time-of-Flight Sensing in Microchannels," IEEE Sens. J., vol. 18, no. 21, pp. 8685-8692, 2018.

[17] H. Berthet, J. Jundt, J. Durivault, B. Mercier, and D. Angelescu, "Time-of-flight thermal flowrate sensor for lab-on-chip applications," Lab Chip, vol. 11, no. 2, pp. 215-223, 2011.

[18] L. V. King, "On the Convection of Heat from Small Cylinders in a Stream of Fluid: Determination of the Convection Constants of Small Platinum Wires, with Applications to Hot-Wire Anemometry," Proc. R. Soc. London A Math. Phys. Eng. Sci., vol. 90, no. 622, pp. 563-570, 1914.

[19] J. S. Gravenstein, M. B. Jaffe, N. Gravenstein, and D. A. Paulus, Capnography, 2nd ed. Cambridge: Cambridge University Press, 2011.

[20] I. Yoshiya, Y. Shimada, and K. Tanaka, "Evaluation for clinical of a hot-wire respiratory flowmeter," J. Appl. Physiol., vol. 47, no. 5, pp. 1131-1135, 1979.

[21] B. Mimoun, A. Van Der Horst, R. Dekker, D. Van Der Voort, A. Van Der Horst, M. Rutten, F. Van De Vosse, and R. Dekker, "Thermal flow sensors on flexible substrates for minimally invasive medical instruments," in IEEE SENSORS, 2012, pp. 3-6.

[22] M. Shikida, J. Naito, T. Yokota, T. Kawabe, Y. Hayashi, and K. Sato, "A catheter-type flow sensor for measurement of aspirated- and inspired-air characteristics in the bronchial region," $J$. Micromechanics Microengineering, vol. 19, no. 10, p. 105027, Oct. 2009.

[23] T. Bork, A. Hogg, M. Lempen, D. Müller, D. Joss, T. Bardyn, P. Büchler, H. Keppner, S. Braun, Y. Tardy, and J. Burger, "Development and in-vitro characterization of an implantable flow sensing transducer for hydrocephalus," Biomed. Microdevices, vol. 12, no. 4, pp. 607-618, 2010.

[24] P. Jiang, S. Zhao, and R. Zhu, "Smart sensing strip using monolithically integrated flexible flow sensor for noninvasively monitoring respiratory flow," Sensors, vol. 15, no. 12, pp. 31738-31750, 2015.

[25] N. Sabaté, J. Santander, L. Fonseca, I. Gràcia, and C. Cané, "Multi-range silicon micromachined flow sensor," Sensors Actuators A Phys., vol. 110, pp. 282-288, 2004.

[26] S. Takagi, "A hot-wire anemometer compensated for ambient temperature variations," J. Phys. E., vol. 19, pp. 739-743, 1986.

[27] C. Sosna, R. Buchner, and W. Lang, "A Temperature Compensation Circuit for Thermal Flow Sensors Operated in Constant-Temperature-Difference Mode," IEEE Trans. Instrum. Meas., vol. 59, no. 6, pp. 1715-1721, Jun. 2010.

[28] R. P. C. Ferreira and R. C. S. Freire, "Hot-wire anemometer with temperature compensation using only one sensor," IEEE Trans. Instrum. Meas., vol. 50, no. 4, pp. 954-958, 2001.

[29] W. Xu, B. Gao, S. Ma, A. Zhang, Y. Chiu, and Y. K. Lee, "Low-cost temperature-compensated thermoresistive micro calorimetric flow sensor by using $0.35 \mu \mathrm{m}$ CMOS MEMS technology," in IEEE Int'l Conf. on Micro Electro Mechanical Systems (MEMS), 2016, pp. 189-192.

[30] G. Kaltsas, P. Katsikogiannis, P. Asimakopoulos, and A. G. Nassiopoulou, "A smart flow measurement system for flow evaluation with multiple signals in different operation modes," Meas. Sci. Technol., vol. 18, p. 3617, 2007.

[31] M. Piotto, M. Dei, F. Butti, G. Pennelli, and P. Bruschi, "Smart flow sensor with on-chip CMOS interface performing offset and pressure effect compensation," IEEE Sens. J., vol. 12, no. 12, pp. 3309-3317, 2012.

[32] G. P. Shen, M. Qin, and Q.-A. Huang, "A cross-type thermal wind sensor with self-testing function," IEEE Sens. J., vol. 10, no. 2, pp. 340-346, 2010.

[33] A. Petropoulos and G. Kaltsas, "Study and Evaluation of a PCB-MEMS Liquid Microflow Sensor," Sensors, vol. 10, no. 10, pp. 8981-9001, Oct. 2010.

[34] W. Xu, B. Lijin, M. Duan, X. Wang, J. Wicaksana, A. Min, M. Ahmed, R. Wang, N. X. Fang, A. Bermak, and Y.-K. Lee, "A wireless dual-mode micro thermal flow sensor system with extended flow range by using CMOS-MEMS process," in IEEE Int'l Conf. on Micro Electro Mechanical Systems (MEMS), 2018, pp. 824-827.

[35] S. Wang, J. Wang, Z. Yi, M. Qir, and Q.-A. Huang, "Experimental Study of a Dual-Mode Control MEMS Wind Sensor with High Accuracy," in IEEE SENSORS, 2018, pp. 31-34.

[36] S. Silvestri and E. Schena, "Micromachined flow sensors in biomedical applications," Micromachines, vol. 3, pp. 225-243, 2012.

[37] R. McCulloch and O. Garcia, "Constant Power Thermal Sensor," US5031126A, 1991

[38] D. Sackett, "Application Note 4470: Constant-Power Source. Maxim Integrated Products," 2009.

[39] V. Kitsos, M. Schormans, A. Demosthenous, and X. Liu, "Asymmetrical Sensing Configuration for Improved Sensitivity in Calorimetric High Flow Measurements in Constant Power Mode," in IEEE SENSORS, 2018, pp. $1-4$.

[40] M. Ahmed, W. Xu, S. Mohamad, F. Boussaid, Y.-K. Lee, and A. Bermak, "Fully Integrated Bidirectional CMOS-MEMS Flow Sensor With Low Power Pulse Operation," IEEE Sens. J., vol. 19, no. 9, pp. 3415-3424, May 2019.

[41] A. Glaninger, A. Jachimowicz, F. Kohl, R. Chabicovsky, and G. Urban, "Wide range semiconductor flow sensors," Sensors Actuators A Phys., vol. 85, no. 1-3, pp. 139-146, Aug. 2000.

[42] Y. Ye, Z. Yi, S. Gao, M. Qin, and Q.-A. Huang, "Effect of Insulation Trenches on Micromachined Silicon Thermal Wind Sensors," IEEE Sens. J., vol. 17, no. 24, pp. 8324-8331, 2017. 